\title{
Ein genetischer Ansatz für die Tourenplanung mit Kundenzeitschranken
}

\section{Stefan Maziejewski, Planungsbüro Transport und Verkehr, Karlsruhe}

Genetische Algorithmen (GA) haben schon oft ihre Qualitäten beim Lösen von klassischen Aufgabenstellungen des Operations Researchs gezeigt. So liegt es nahe, auch Probleme aus der Praxis mit dieser Algorithmenklasse anzugehen. In diesem Vortrag wird ein GA vorgestellt, der für Tourenplanungsprobleme mit Kundenzeitschranken gute Lösungen generiert. Die Ergebnisse sind in der Regel deutlich besser als die vom Savingsalgorithmus erzeugten Resultate. Tourenplanungsprobleme aus der Literatur von Padberg und Rinaldi konnten um bis zu 2,7\% verbessert werden.

Die genetischen Algorithmen arbeiten nach folgender Vorgehensweise: zunächst wird mittels einer einfachen Heuristik eine Population aus Individuen erzeugt. Jeweils zwei Individuen aus der Population werden selektiert und mittels eines Kreuzungsoperators ein Nachkomme generiert. Dieser Nachkomme wird lokal optimiert und falls er gut genug ist, in die Population aufgenommen. Von Zeit zu Zeit mutieren einige Individuen der Population.

In der Arbeit werden verschiedene Algorithmen für die einzelnen genetischen Operatoren (Selektion, Kreuzung, Nachoptimierung und Mutation) vorgeschlagen, die speziell auf die Aufgabenstellung angepaßt sind. Diese werden mit verschiedenen Parametern (Populationsgröße, maximale Lebensdauer, Mutationsrate) getestet und verglichen. Umfangreiche Testläufe zeigen, daß eine ausgewogene Kombination von Zufall und gezielter Optimierung die besten Resultate liefert. 